\title{
Existence of local and global solutions of fuzzy delay differential inclusions
}

\author{
Chao Min $^{1,2^{*}}$, Nan-jing Huang ${ }^{3}$ and Lie-Hui Zhang ${ }^{1}$
}

\author{
*Correspondence: \\ minchaosc@126.com \\ 'State Key Laboratory of Oil and Gas \\ Reservoir Geology and Exploitation, \\ Southwest Petroleum University, \\ Xindu Road 8 in Xindu District, \\ Chengdu, China \\ ${ }^{2}$ School of Science, Southwest \\ Petroleum University, Xindu Road 8 \\ in Xindu District, Chengdu, China \\ Full list of author information is \\ available at the end of the article
}

\begin{abstract}
Fuzzy delay differential inclusions are introduced and studied in this paper. The local and global existence theorems under different conditions are proved by using selection theorems and Kakutani's fixed point theorem. Under the tangential condition, a global viable solution for a fuzzy delay differential inclusion is proved to exist. The property of the solution sets is achieved. Some known results of fuzzy differential inclusions and fuzzy differential equations are extended, which might be helpful in the analysis of dynamic systems with uncertainties.
\end{abstract}

Keywords: fuzzy delay differential inclusion; fuzzy differential equation; fixed point theorem; selection theorem

\section{Introduction}

It is an effective way to describe the performance of a system with uncertainties to use fuzzy differential equations (FDEs). In recent years, the study of FDEs has received much attention and there are many works having been done for the existence and uniqueness of solutions of FDEs under different conditions, such as [1-9] etc. Within the references, there are several methods to discuss the FDEs, as follows.

(a) The first approach is using the Hukuhara derivative of a fuzzy valued function, but in this framework the diameter of the solution $x(t)$ of some FDE is unbounded as the time tincreases [10], which is quite different from the crisp cases.

(b) The second approach is presented by Hüllermeier [11]. He replaced the FDE by a family of differential inclusions, which overcame the preceding problem. However, this method is still not ideal; the solutions may not be fuzzy valued maps. This idea was developed by Lakshmikantham et al. [12,13]. Combining with the idea of Aubin $[14,15]$, this approach was exploited by Diamond in $[10,16]$ where the assumption of fuzzy convexity and the compactness of level sets are removed.

(c) The third approach is to generalize the differentiability of fuzzy valued functions. In [7, 17], Bede et al. studied the fuzzy initial valued (FIVP) and 2-point boundary value problems by defining a generalized differentiability, which allows to obtain the solutions of fuzzy differential equations and these solutions may have decreasing diameters. Unexpectedly, there are usually 2 solutions for the FIVP with respect to this derivative. The work of [18-21] is mainly based on this idea.

(d) The fourth approach is by applying a parametric representation of fuzzy numbers. In [22, 23], Chen et al. established a new definition for differentiability of fuzzy valued

\section{Springer}

(c)2014 Min et al.; licensee Springer. This is an Open Access article distributed under the terms of the Creative Commons Attribution License (http://creativecommons.org/licenses/by/2.0), which permits unrestricted use, distribution, and reproduction in any medium, provided the original work is properly cited. 
functions and proved the existence and uniqueness of the solutions for the 2-point boundary value problems.

(e) The fifth approach was started by Liu's pioneering paper Fuzzy process, hybrid process and uncertain process [24], in which the fuzzy differential equation is regarded as a type of differential equations driven by Liu process, similar to the stochastic differential equation. Thereafter, this method was introduced into option pricing for fuzzy financial markets [25] and fuzzy optimal control with application to portfolio selections [26].

In 2011, Choudary and Donchev [27] pointed out that the proof of the celebrated theorem of Nieto [28] is not true, which makes it still an open question under what right-hand side conditions the fuzzy differential equation has a solution. As is well known, it is a useful method to treat the differential equations with weakened right-hand side by differential inclusions [29].

On the other hand, it is meaningful to study the FDEs through differential inclusions in fuzzy control theory. Given a fuzzy control system $x^{\prime}(t)=f(t, x(t), u(t))$, although $f$ is fuzzy valued, to obtain a viable trajectory $x(t)$, the control $u(t)$ still have to be crisp despite the uncertainty of the system for the sake of realizability. Thus it is not enough to discuss the FDEs just through the fuzzy differentiability.

Moreover, most of the discussions on the qualitative problems of FDEs are based on the idea of Hüllermeier. Therefore, discussing the solutions of fuzzy differential inclusions is worth trying in the study of FDEs.

Fuzzy differential inclusions (FDIs) were first presented by Baidosov [30]. Aubin [15] and Dordan [31] discussed the viability of the FDIs in an equivalent form with toll sets. Lakshmikantham and Mohapatra [13] presented a theorem to show under what conditions the attainable sets of FDIs are the level sets of a fuzzy map. In 2000, Zhu and Rao [32] presented two types of FDIs as follows:

$$
\left\{\begin{array}{l}
F_{(t, x(t))}\left(x^{\prime}(t)\right)>(\text { or } \geq) \alpha(x(t)) \\
\quad \text { i.e. } x^{\prime}(t) \in\left(F_{(t, x(t))}\right) \alpha_{\alpha(x(t))}\left(\text { or }\left[F_{(t, x(t))}\right]_{\alpha(x(t)))}\right) \\
x\left(t_{0}\right)=x_{0}
\end{array}\right.
$$

where the existence of the solutions is proved for the open and the closed situation, respectively. FDI is actually a type of parameterized differential inclusions, thus Zhu's results extended the theory of FDIs to time-varying case by turning $\alpha$ into $\alpha(x(t))$.

In dynamic systems, delay is generally inevitable, that is, the velocity of the system at some instant depends on the history of the trajectory until this instant. In [33], Lupulescu applied a successive approximation method to discuss the fuzzy differential equations with distributed delays. Guo et al. [34] discussed the oscillation properties of a class of fuzzy delay differential equation of second order and provided an oscillation criterion. Malinowski [35] took the randomness into consideration and discussed the stochastic fuzzy delayed differential equations in the modeling of population growth. In [36], Kloeden and Lorenz removed the assumption of fuzzy convexity of FDEs and discussed the fuzzy delay differential equations (FDDEs) in this perspective.

In this paper, Zhu's idea is extended to the fuzzy delay differential inclusions (FDDIs), which will help describe the dynamic systems with uncertainties and delays. The existence and the properties of the solutions of FDDIs are discussed and will help enrich the theory of FDEs. 


\section{Preliminaries}

Definition 2.1 A fuzzy subset $A$ of $\mathbf{R}^{n}$ is defined with its membership function, $\mu_{A}: \mathbf{R}^{n} \rightarrow$ $[0,1]$. Then the fuzzy set $A$ could be rewritten as $\left\{\left(x, \mu_{A}\right): x \in \mathbf{R}^{n}\right\}$. The $\alpha$-level set of $A$ is denoted by

$$
[A]_{\alpha}=\left\{x: \mu_{A}(x) \geq \alpha\right\}
$$

for $\alpha \in(0,1]$, and $[A]_{0}$ is defined as $[A]_{0}=\overline{\left\{x \in \mathbf{R}^{n}: A(x)>0\right\}}$. Moreover,

$$
(A)_{\alpha}=\left\{x: \mu_{A}(x)>\alpha\right\}
$$

is said to be the $\alpha$-open level set of $A$ for $\alpha \in[0,1)$.

We do not distinguish a fuzzy set $A$ and its membership function $\mu_{A}$, that is, the membership of $x \in \mathbf{R}^{n}$ could be simply written as $A(x)$. Denote the family of all the fuzzy sets of a space $X$ by $\mathcal{F}(X)$.

Denote $\mathbf{E}^{n}=\left\{u: \mathbf{R}^{n} \rightarrow[0,1], u\right.$ satisfies (1)-(4) below $\}$, where

(1) $u$ is normal, that is, there exists an $x \in \mathbf{R}^{n}$ such that $u\left(x_{0}\right)=1$;

(2) $u$ is fuzzy convex, that is, for any $x, y \in \mathbf{R}^{n}$ and $\lambda \in[0,1]$,

$u(\lambda x+(1-\lambda) y) \geq \min \{u(x), u(y)\}$, which is equivalent with that for any $\alpha \in[0,1]$,

$[u]_{\alpha}=\{x: u(x) \geq \alpha\}$ is a convex set;

(3) $u$ is upper semicontinuous, that is, for any $\alpha,[u]_{\alpha}$ is a closed set;

(4) $[u]_{0}=\overline{\left\{x \in \mathbf{R}^{n}: u(x)>0\right\}}$ is compact.

It is obvious that, for each $u \in \mathbf{E}^{n}$, the $\alpha$-level sets $[u]_{\alpha}$ are convex compact subsets of $\mathbf{R}^{n}$ for all $\alpha \in[0,1]$.

Remark 2.2 We should notice that a fuzzy valued map $F: X \rightarrow \mathbf{E}^{n}$ can generate a real valued function $\widetilde{F}: X \times \mathbf{R}^{n} \rightarrow[0,1]$, where for any $x \in X, y \in \mathbf{R}^{n}, \widetilde{F}(x, y)=F(x)(y)$. For convenience we do not distinguish $F$ and $\widetilde{F}$ in the following discussion, and we denote them both by $F$.

A set-valued map $F: X \rightarrow Y$ is strict, if its domain $\operatorname{Dom}(F)=\{x \in X \mid F(x) \neq \emptyset\}=X$. By the definition of $\mathbf{E}^{n}$, we can see that for any fixed $x \in X$, the function $F(x, \cdot): \mathbf{R}^{n} \rightarrow[0,1]$ is strict, convex, and upper semicontinuous. Moreover, the closed set $\left\{y \in \mathbf{R}^{n}: F(x, y) \geq \alpha\right\}$ is compact for any $\alpha \in[0,1]$.

Denoting the family of compact subsets of $\mathbf{R}^{n}$ by $P_{k c}\left(\mathbf{R}^{n}\right)$, for any $A, B \in P_{k c}\left(\mathbf{R}^{n}\right)$, the Hausdorff metric of $P_{k c}\left(\mathbf{R}^{n}\right)$ is

$$
H(A, B)=\inf \{\varepsilon>0 \mid A \subset V(B, \varepsilon), B \subset V(A, \varepsilon)\} .
$$

Then one can define the metric of $\mathbf{E}^{n}$ as follows, for any $\phi, \psi \in \mathbf{E}^{n}$ :

$$
D(\phi, \psi)=\sup _{\alpha \in[0,1]} H\left([\phi]_{\alpha},[\psi]_{\alpha}\right) .
$$

Thus, $\left(\mathbf{E}^{n}, D\right)$ is a complete metric space. 
Definition 2.3 (i) A set-valued map $F: X \rightarrow Y$ is called upper semicontinuous at $x \in X$ if and only if for any neighborhood $U$ of $F(x)$, there exists $\eta>0$ such that $F\left(x^{\prime}\right) \subset U$ for all $x^{\prime} \in B_{X}(x, \eta)$. A set-valued map $F$ is said to be upper semicontinuous if and only if it is upper semicontinuous at any point of $X$.

(ii) A set-valued map $F$ is lower semicontinuous at $x \in \operatorname{Dom}(F)$ if and only if for any $y \in F(x)$ and for any sequence of elements $x_{n} \in \operatorname{Dom}(F)$ converging to $x$, there exists a sequence of elements $y_{n} \in F\left(x_{n}\right)$ converging to $y$. A set-valued map $F$ is said to be lower semicontinuous if it is lower semicontinuous at every point $x \in \operatorname{Dom}(F)$.

Contingent cones and contingent derivatives are important tools in discussing the existence of the solution of differential inclusions. Let $K$ be a nonempty set in a Hilbert space, the contingent cone $T_{K}(x)$ to $K$ at $x$ is defined as

$$
v \in T_{k}(x) \Longleftrightarrow \liminf _{h^{+} \rightarrow 0} \frac{d_{K}(x+h v)}{h}=0,
$$

where $d_{K}(x+h v)$ is the distance from $x+h v$ to $K$. Let $F$ be a strict set-valued map from $X$ to $Y$ and $\left(x_{0}, y_{0}\right)$ belong to the graph of $F$. We denote by $D F\left(x_{0}, y_{0}\right)$ the set-valued map whose graph is the contingent cone $T_{\operatorname{graph}(F)}\left(x_{0}, y_{0}\right)$ to the graph of $F$ at $\left(x_{0}, y_{0}\right)$, i.e.,

$$
v_{0} \in D F\left(x_{0}, y_{0}\right)\left(u_{0}\right) \quad \Longleftrightarrow \quad\left(u_{0}, v_{0}\right) \in T_{\operatorname{graph}(F)}\left(x_{0}, y_{0}\right) .
$$

Definition 2.4 We say that the set-valued map $D F\left(x_{0}, y_{0}\right)$ from $X$ to $Y$ is the contingent derivative of $F$ at $x_{0} \in K$ and $y_{0} \in F\left(x_{0}\right)$.

Given any $\tau \geq 0$, we let $C_{0}=C\left[[-\tau, 0], \mathbf{R}^{n}\right]$. For any $\phi \in C_{0}$, the norm of $\phi$ is defined as

$$
\|\phi\|=\sup _{-\tau \leq s \leq 0}\|\phi(s)\| .
$$

Thus for any $\phi, \psi \in C_{0}$, the metric on $C_{0}$ is

$$
D[\phi, \psi]=\sup _{-\tau \leq s \leq 0}\|\phi(s)-\psi(s)\| .
$$

Suppose that $x \in C=C\left[J_{0}, \mathbf{R}^{n}\right]$, where $J_{0}=\left[t_{0}-\tau, t_{0}+a\right]$ with $a>0$. For any $t \geq t_{0}, t \in J_{0}$, we denote the translation of the restriction of $x$ to the interval $[t-\tau, t]$ by $x_{t}$, that is, $x_{t} \in C_{0}$ and

$$
x_{t}(s)=x(t+s), \quad-\tau \leq s \leq 0,
$$

which means that the graph of $x_{t}$ is actually the graph of $x$ on $[t-\tau, t]$ shifted to $[-\tau, 0]$. Let $\Omega$ be an open set of $\mathbf{R} \times C_{0}, F: \Omega \rightarrow \mathbf{E}^{n}$ be a fuzzy valued map, $\left(t_{0}, \phi\right) \in \Omega$. We consider the fuzzy differential inclusions with delay as follows:

$$
\left\{\begin{array}{l}
x^{\prime}(t) \in\left[F\left(t, x_{t}\right)\right]_{\alpha(x)}, \\
x_{t_{0}}=\phi .
\end{array}\right.
$$

In the following section, we mainly discuss the existence of the solution of (1). We should notice that if $\tau=0$, the existence of the solutions of FDDIs above is restricted to the results of Zhu and Rao [32]. 


\section{Local existence of the solutions of FDDIs}

First of all, we shall apply some selection theorems to discuss the solutions of FDDIs under different conditions. The key point is to discuss the continuity of $F$ when $\alpha$ is time varying.

Lemma 3.1 (Michael's selection theorem [37]) Let X be a metric space, $Y$ a Banach space. Let $F$ from $X$ into the closed convex subsets of $Y$ be lower semicontinuous. Then there exists $f: X \rightarrow Y$, a continuous selection from $F$.

Theorem 3.2 Let $\Omega$ be an open subset in $\mathbf{R} \times C_{0}$ with $\left(t_{0}, \phi\right) \in \Omega$. Suppose that $F: \Omega \rightarrow \mathbf{E}^{n}$ is a fuzzy map, the corresponding function $F(t, x, y): \Omega \times \mathbf{R}^{n} \rightarrow[0,1]$ of which is continuous at $(t, x) \in \Omega$ and differentiable at $y \in \mathbf{R}^{n}$. Let $\alpha: \mathbf{R}^{n} \rightarrow(0,1]$ be an upper semicontinuous function. Assume that the transversality condition is satisfied, that is, for any $\left(t_{0}, \phi\right) \in \Omega$ and $y_{0} \in \mathbf{R}^{n}$ there exist constants $c>0$ and $\eta>0$ such that

$$
\left\{\begin{array}{l}
\forall(t, x) \in B\left(\left(t_{0}, \phi\right), \eta\right), y \in B\left(y_{0}, \eta\right), z \in B\left(F\left(t_{0}, \phi, y_{0}\right)\right) \text { and } z \geq \alpha(x) \\
\text { the unit ball of } \mathbf{R}, B_{R} \subset c F_{y}^{\prime}\left(t, x, B_{\mathbf{R}^{n}}\right)-[a(x)-z,+\infty)
\end{array}\right.
$$

Then on some $I=\left[t_{0}-\tau, t_{0}+T\right]$, there exists a continuous differentiable function $x: I \rightarrow \mathbf{R}^{n}$, which is the solution of FDDI (1).

Proof We define a set-valued map $\widetilde{F}: \Omega \rightarrow \mathbf{R}^{n}$ by

$$
\widetilde{F}(t, x)=\left\{y \in \mathbf{R}^{n}: F(t, x, y) \geq \alpha(x)\right\}, \quad(t, x) \in \Omega .
$$

As the value of $F$ belongs to $\mathbf{E}^{n}$ and $\alpha(x) \in[0,1), \widetilde{F}(t, x)$ is always nonempty. For any $u, v \in \widetilde{F}(t, x)$ and $\lambda \in[0,1]$, by the convexity of $F(t, x) \in \mathbf{E}^{n}$, we have

$$
F(t, x)(\lambda u+(1-\lambda) v) \geq \min \{F(t, x)(u), F(t, x)(v)\} \geq \alpha(x),
$$

and so $\lambda u+(1-\lambda) v \in \widetilde{F}(t, x)$, which means that $\widetilde{F}(t, x)$ is convex for each $(t, x) \in \Omega$.

Next we prove that $\widetilde{F}(t, x)$ is lower semicontinuous. Define a set-valued map $G(t, x)$ : $\Omega \rightarrow \mathbf{R}$ by

$$
G(t, x)=\{r: r \geq \alpha(x)\}=[\alpha(x), \infty) .
$$

Obviously, $G(t, x)$ is closed. We show that $G(t, x)$ is lower semicontinuous. For any $r \in$ $G(t, x)$, sequence $\left\{\left(t_{n}, x_{n}\right)\right\}$ converging to $(t, x)$, we need to find $r_{n} \in G\left(t_{n}, x_{n}\right)$ such that $r_{n} \rightarrow r$. As $\alpha(x)$ is upper semicontinuous and $x_{n} \rightarrow x$, we know that $\lim _{\sup _{n \rightarrow \infty}} \alpha\left(x_{n}\right) \leq$ $\alpha(x) \leq r$. It is obvious that there exist at most a finite number of $\alpha\left(x_{n}\right)$ that might be greater than $r$. Denote by $N$ the max index of $\alpha\left(x_{n}\right)$ greater than 1 and $e_{N}$ the difference between the $\max \alpha\left(x_{n}\right)$ and $r$. Let

$$
r_{n}= \begin{cases}r+e_{n}, & n \leq N, \\ r+\frac{1}{n}, & n>N .\end{cases}
$$

Then $\left\{r_{n}\right\}$ is the required sequence. 
Notice that for any $z \in G(t, x), T_{G(t, x)}(z)=[\alpha(x)-z,+\infty)$, the transversality condition turns out to be

$$
\left\{\begin{array}{l}
\forall(t, x) \in B\left(\left(t_{0}, \phi\right), \eta\right), y \in B\left(y_{0}, \eta\right), z \in B\left(F\left(t_{0}, \phi, y_{0}\right)\right) \cap G(t, x) \\
\text { the unit ball of } \mathbf{R}, B_{R} \subset c F_{y}^{\prime}\left(t, x, B_{\mathbf{R}^{n}}\right)-T_{G(t, x)}(z)
\end{array}\right.
$$

From Theorem 1.5.5 in [38], we get

$$
\widetilde{F}(t, x)=\left\{y \in \mathbf{R}^{n}: F(t, x, y) \geq \alpha(x)\right\}=\left\{y \in \mathbf{R}^{n}: F(t, x, y) \in G(t, x)\right\}
$$

is lower semicontinuous.

By Lemma 3.1, there exists a continuous selection $f\left(t, x_{t}\right) \in \widetilde{F}\left(t, x_{t}\right)$. Thus, it is enough to prove the existence of the solution of the following initial value problem with delay (DIVP):

$$
\left\{\begin{array}{l}
x^{\prime}(t)=f\left(t, x_{t}\right), \\
x_{t_{0}}=\phi .
\end{array}\right.
$$

This is a classical result, one can find the proof in any book concerned with functional differential equations, like [39]. This completes the proof.

The next theorem is about the FDDIs in the open case based on Zhu's idea, that is,

$$
\left\{\begin{array}{l}
x^{\prime}(t) \in\left(F\left(t, x_{t}\right)\right)_{\alpha(x(t))}, \\
x_{t_{0}}=\phi
\end{array}\right.
$$

where

$$
\left(F\left(t, x_{t}\right)\right)_{\alpha(x)}=\left\{y \in \mathbf{R}^{n}: F\left(t, x_{t}, y\right)>\alpha(x)\right\} .
$$

We should notice that $\widetilde{F}(t, x)=\left\{y \in \mathbf{R}^{n}: F(t, x, y)>\alpha(x)\right\}$ is not closed valued, which means that even if the set-valued map $\widetilde{F}$ is lower semicontinuous, Michael's selection theorem still cannot be applied in this situation. To get a solution of (2), the continuous selection theorem of Yannelis and Prabhakar [40] is employed in this paper.

Lemma 3.3 ([40]) Let D be a paracompact Hausdorff topological space, $Y$ be a topological vector space and $F: D \rightarrow 2^{Y}$ be a multifunction with nonempty convex values. If $F$ has open lower sections, that is, for any $y \in Y, F^{-1}(y)=\{x \in D \mid y \in F(x)\}$ is open in $D$, then there exists a continuous function $f: D \rightarrow Y$ such that $f(x) \in F(x)$ for any $x \in D$.

Theorem 3.4 Let $\Omega$ be an open subset in $\mathbf{R} \times C_{0}$ with $\left(t_{0}, \phi\right) \in \Omega$. Suppose that $F: \Omega \rightarrow \mathbf{E}^{n}$ is a fuzzy map, the corresponding function $F(t, x, y): \Omega \times \mathbf{R}^{n} \rightarrow[0,1]$ of which is lower semicontinuous at $(t, x) \in \Omega$. Let $\alpha: \mathbf{R}^{n} \rightarrow[0,1)$ be an upper semicontinuous function. Then on some $I=\left[t_{0}-\tau, t_{0}+T\right]$, there exists a continuous differentiable function $x: I \rightarrow \mathbf{R}^{n}$, which is a solution of the open FDDI (2).

Proof We define a set-valued map $\widetilde{F}: \Omega \rightarrow \mathbf{R}^{n}$ by

$$
\widetilde{F}(t, x)=\left\{y \in \mathbf{R}^{n}: F(t, x, y)>\alpha(x)\right\}, \quad(t, x) \in \Omega .
$$


As the values of $F$ belongs to $\mathbf{E}^{n}$ and $\alpha(x) \in[0,1), \widetilde{F}(t, x)$ is always nonempty. For any $u, v \in$ $\widetilde{F}(t, x)$ and $\lambda \in[0,1]$, by the convexity of $F(t, x) \in \mathbf{E}^{n}$, we have

$$
F(t, x)(\lambda u+(1-\lambda) v) \geq \min \{F(t, x)(u), F(t, x)(v)\}>\alpha(x)
$$

and so $\lambda u+(1-\lambda) v \in \widetilde{F}(t, x)$, which means that $\widetilde{F}(t, x)$ is convex for each $(t, x) \in \Omega$.

For any $y \in \mathbf{R}^{n}$, we assert that $\widetilde{F}^{-1}(y)=\{(t, x) \in \Omega, F(t, x, y)>\alpha(x)\}$ is open in $\mathbf{R}^{n}$. It is sufficient to verify that $\left(\widetilde{F}^{-1}(y)\right)^{c}=\{(t, x) \in \Omega, F(t, x, y) \leq \alpha(x)\}$ is closed. In fact, for any $\left\{\left(t_{n}, x_{n}\right)\right\} \subset\left(\widetilde{F}^{-1}(y)\right)^{c}$, we have $F\left(t_{n}, x_{n}, y\right) \leq \alpha\left(x_{n}\right)$. Let $\left(t_{n}, x_{n}\right) \rightarrow(t, x)$ as $n \rightarrow \infty$. By the lower semicontinuity of $F(\cdot, \cdot, y)$ and the upper semicontinuity of $\alpha(\cdot)$, we have

$$
F(t, x, y) \leq \liminf _{n \rightarrow \infty} F\left(t_{n}, x_{n}, y\right) \leq \limsup _{n \rightarrow \infty} \alpha\left(x_{n}\right) \leq \alpha(x)
$$

and so $(t, x) \in\left(\widetilde{F}^{-1}(y)\right)^{c}$. Thus, $\left(\widetilde{F}^{-1}(y)\right)^{c}$ is closed and so $\widetilde{F}$ is a nonempty convex set-valued map with open lower sections. By Lemma 3.3, there exists a continuous selection $f: \Omega \rightarrow$ $\mathbf{R}^{n}$ of $\widetilde{F}$ such that, for each $\left(t, x_{t}\right) \in \Omega, f\left(t, x_{t}\right) \in \widetilde{F}\left(t, x_{t}\right)$. Then the proof of the existence of the solution of (2) is the same as Theorem 3.2. This completes the proof.

Remark 3.5 Theorems 3.2 and 3.2 extend Theorems 1 and 3 of Zhu and Rao [32] to the cases with delay, respectively.

When $\alpha(x)$ is lower semicontinuous, the selection theorems cannot be applied in the above theorems. To get the solution of (1), we employ Kakutani's fixed point theorem and convergence theorem.

Lemma 3.6 (Kakutani's fixed point theorem [41]) Let $K$ be a compact convex subset of a Banach space $X$ and let $F$ be an upper semicontinuous map from $K$ into its compact convex subsets. Then $F$ has a fixed point, i.e. there exists $\bar{x}$ belonging to $F(\bar{x})$.

Lemma 3.7 (Convergence theorem [41]) Let F be a proper upper hemicontinuous map from a Hausdorff locally convex space $X$ to the closed convex subsets of a Banach space $Y$. Let $I$ be an interval of $\mathbf{R}$ and $x_{k}(\cdot)$ and $y_{k}(\cdot)$ be measurable functions from $I$ to $X$ and $Y$, respectively, satisfying, for almost all $t \in I$, that for every neighborhood $\mathcal{N}$ of 0 in $X \times Y$ there exists $k_{0} \doteq k_{0}(t, \mathcal{N})$ such that $\forall k \geq k_{0},\left(x_{k}(t), y_{k}(t)\right) \in \operatorname{graph}(F)+\mathcal{N}$. If

(i) $x_{k}(\cdot)$ converges almost everywhere to a function $x(\cdot)$ from I to $X$,

(ii) $y_{k}(\cdot)$ belongs to $L^{1}(I, Y)$ and converges weakly to $y(\cdot)$,

then for almost all $t \in I$

$$
(x(t), y(t)) \in \operatorname{graph}(F), \quad \text { i.e. } y(t) \in F(x(t)) .
$$

Based on the above two lemmas, we can get the following existence theorem of the solutions of FDDI (1).

Theorem 3.8 Let $\Omega$ be an open subset in $\mathbf{R} \times C_{0}$ with $\left(t_{0}, \phi\right) \in \Omega$. Suppose that $F: \Omega \rightarrow \mathbf{E}^{n}$ is a fuzzy map, the corresponding function $F(t, x, y): \Omega \times \mathbf{R}^{n} \rightarrow[0,1]$ of which is upper semicontinuous at $(t, x) \in \Omega$. Let $\alpha: \mathbf{R}^{n} \rightarrow[0,1)$ be a lower semicontinuous function. Moreover, 
if there exists a neighborhood $D$ of $\left(t_{0}, \phi\right)$ such that $\bigcup_{(t, x) \in D}[F(t, x)]_{\alpha(x)}$ is compact in $\mathbf{R}^{n}$, then on some $I=\left[t_{0}-\tau, t_{0}+\alpha\right]$, there exists an absolutely continuous function $x: I \rightarrow \mathbf{R}^{n}$, which is a solution of problem (1).

Proof Define a set-valued map $\widetilde{F}(t, x)$ as follows:

$$
\widetilde{F}(t, x)=\left\{y \in \mathbf{R}^{n}: F(t, x, y) \geq \alpha(x)\right\} .
$$

We claim that $\widetilde{F}(t, x)$ is an upper semicontinuous set-valued map on $D$ with nonempty compact convex values. By the definition of $\mathbf{E}^{n}$, it is clear that $\widetilde{F}(t, x)$ is nonempty, compact and convex in $\mathbf{R}^{n}$. Since $\mathbf{R} \times \mathbf{R}^{n}$ is locally compact, for each compact subset $K \subset D$, the graph of the restriction of $\widetilde{F},\left.\widetilde{F}\right|_{K}: K \rightarrow \mathbf{R}^{n}$ is closed. Actually for any Cauchy sequence $\left(t_{n}, x_{n}, y_{n}\right) \subset \operatorname{graph}\left(\left.\widetilde{F}\right|_{K}\right)$ which converges to $(t, x, y)$, by the upper semicontinuity of $F(t, x, y)$ and lower semicontinuity of $\alpha(x)$, we have

$$
F(t, x, y) \geq \limsup _{n \rightarrow \infty} F\left(t_{n}, x_{n}, y_{n}\right) \geq \liminf _{n \rightarrow \infty} \alpha\left(x_{n}\right) \geq \alpha(x) .
$$

Therefore, $(t, x, y) \in \operatorname{graph}\left(\left.\widetilde{F}\right|_{K}\right)$ and so $\operatorname{graph}\left(\left.\widetilde{F}\right|_{K}\right)$ is a closed subset of $K \times \bigcup_{(t, x) \in D}[F(t$, $x)]_{\alpha(x)}$. Thus, $\operatorname{graph}\left(\left.\widetilde{F}\right|_{K}\right)$ is also compact and so $\widetilde{F}(t, x)$ is upper semicontinuous on $D$.

Because of the compactness of $\bigcup_{(t, x) \in D}[F(t, x)]_{\alpha(x)}$, we know that $\widetilde{F}(t, x)$ is bounded in $D$, that is, there exists some $M$ such that

$$
\|\widetilde{F}(t, \phi)\|:=\sup _{y \in \widetilde{F}(t, \phi)}\|y\| \leq M, \quad \forall(t, \phi) \in D .
$$

Moreover, there must be some $\alpha \leq a$ and $\beta$ such that, for any $t \in[0, \alpha]$,

$$
y \in \mathcal{A}(\alpha, \beta):=\left\{y \in C\left([-\tau, \alpha], \mathbf{R}^{n}\right): y_{0}=\phi, y_{t} \in C_{0} \text { and }\left\|y_{t}\right\| \leq \beta\right\}
$$

This shows that $\left(t_{0}+t, y_{t}+\phi_{t_{0}+t}\right) \in D$.

Define a set-valued map $T: \mathcal{A}(\alpha, \beta) \rightarrow 2^{C\left([-\tau, \alpha], \mathbf{R}^{n}\right)}$ as follows:

$$
T(y)=\left\{u \in C\left([-\tau, \alpha], \mathbf{R}^{n}\right): u(t)=\left\{\begin{array}{l}
\phi(t), t \in[-\tau, 0], \\
\phi(0)+\int_{0}^{t} f(s) d s, t \in[0, \alpha], f(t) \text { is the } \\
\text { integrable selection of } \widetilde{F}\left(t_{0}+t, y_{t}+\phi_{t_{0}+t}\right.
\end{array}\right\} .\right.
$$

As $\widetilde{F}$ is upper semicontinuous with closed values, there exists integrable selection of $\widetilde{F}$. Thus $T$ has nonempty values. Now we will verify that $T$ is an upper semicontinuous setvalued map with compact and convex values. For any $y \in \mathcal{A}(\alpha, \beta)$ and $u(t) \in T(y), \widetilde{F}\left(t_{0}+\right.$ $\left.t, \phi_{t_{0}+t}+y_{t}\right)$ has an integrable selection $f(t)$, such that

$$
u(t)=\phi(0)+\int_{0}^{t} f(s) d s, \quad t \in[0, \alpha]
$$

For any $t, r \in[0, \alpha]$, we have

$$
\begin{aligned}
\|u(t)-u(r)\| & \leq\left\|\int_{r}^{t} f(s) d s\right\| \leq\left\|\int_{r}^{t} \widetilde{F}\left(t_{0}+t, \phi_{t_{0}+t}+y_{t}\right) d s\right\| \\
& \leq M(t-r) .
\end{aligned}
$$


Let $K=\left\{u \in C\left([-\tau, \alpha], \mathbf{R}^{n}\right):\|u(t)-u(r)\| \leq M(t-r)\right.$ and $\left.\|u(t)\| \leq M \alpha, \forall t, r \in[0, \alpha]\right\}$. Then $K$ is a compact subset of $C([-\tau, \alpha])$. If $M \alpha<\beta$, we know that $T(y) \subset K$ and $K \subset \mathcal{A}(\alpha, \beta)$. As $T(y)$ is the subset of a compact set $K$, to verify the upper semicontinuity of $T$, it is enough to prove that the graph of $T$ is closed.

For any $\left(y_{k}, u_{k}\right) \in \operatorname{graph}(T)$, and $\left(y_{k}, u_{k}\right) \rightarrow(y, u)$, there exists an integrable selection $f_{k}(t)$ of $\widetilde{F}\left(t_{0}+t, \phi_{t_{0}+t}+y_{k, t}\right)$ such that $u_{k}(t)=\int_{0}^{t} f_{k}(s) d s$. For any $t \in[0, \alpha]$, we have $\left\|f_{k}(t)\right\| \leq$ $\left\|\widetilde{F}\left(t_{0}+t, \phi_{t_{0}+t}+y_{k, t}\right)\right\| \leq M$ and so $\left\{f_{k}\right\}$ is contained in a ball of $L^{\infty}\left([0, \alpha], \mathbf{R}^{n}\right)$. By Alaoglu's theorem, $\left\{f_{k}\right\}$ has a weak* convergent subsequence, without loss of generality, denoted still by $\left\{f_{k}\right\}$. Suppose that $\left\{f_{k}\right\} \rightarrow f$. For any $g \in L^{\infty}\left([0, \alpha], \mathbf{R}^{n}\right)$, we have

$$
\int_{0}^{\alpha}\left\langle f_{k}(s), g(s)\right\rangle d s \rightarrow \int_{0}^{\alpha}\langle f(s), g(s)\rangle d s
$$

and so $\int_{0}^{t} f_{k}(s) d s \rightarrow \int_{0}^{t} f(s) d s$ for any $t \in[0, \alpha]$. Since $u_{k}=\phi(0)+\int_{0}^{t} f_{k}(s) d s$, we know that $u(t)=\phi(0)+\int_{0}^{t} f(s) d s$. For any neighborhood $\mathcal{N}$ of $0 \in \mathcal{A}(\alpha, \beta) \times \mathbf{R}^{n}$, there exists a constant $k_{0}$ such that

$$
\left(y_{k}, f_{k}\right) \in \operatorname{graph}(\widetilde{F})+\mathcal{N}, \quad \forall k \geq k_{0} .
$$

By Lemma 3.7, we have $f(t) \in \widetilde{F}\left(t_{0}+t, \phi_{t_{0}+t}+y_{k, t}\right)$ and so the graph of $T$ is closed. This shows that $T$ is upper semicontinuous. By the compactness of $K$ and the convexity of $\widetilde{F}$, it is easy to see that $T$ is an upper semicontinuous set-valued map with nonempty convex compact values. It follows from Lemma 3.6 that $T$ has a fixed point in $K$, which is the solution of FDDIs (1). This completes the proof.

\section{Global existence of the solutions of FDDIs}

In this section, we extend the existence results presented in Section 3 to the global situation. For later discussion, we first extend the comparison theorems of Lakshmikantham and Mohapatra [13] to fuzzy differential inclusions. The proof is based on the same routine as Theorem 3.4.1 of [13]. Let $J=\left[t_{0}, t_{0}+a\right]$ and $\mathbf{R}_{+}=[0,+\infty)$.

Lemma 4.1 Assume that for a fuzzy valued map $F: J \times \mathbf{R}^{n} \rightarrow \mathbf{E}^{n}, \forall t \in J, u, v \in \mathbf{R}^{n}$,

$$
D(F(t, u), F(t, v)) \leq g(t, D(u, v))
$$

where $g \in C\left[J \times \mathbf{R}_{+}, \mathbf{R}_{+}\right]$and $g(t, w)$ is nondecreasing in $w$ for each $t$. Suppose that the maximal solution $r\left(t, t_{0}, w\right)$ of the scalar differential equation

$$
w^{\prime}=g(t, w), \quad w\left(t_{0}\right)=w_{0} \geq 0,
$$

exists on $J$. Then, if $u(t), v(t)$ are any two solutions of

$$
x^{\prime}(t) \in[F(t, x)]_{\alpha(x)}, \quad x\left(t_{0}\right)=x_{0},
$$

through $\left(t_{0}, u_{0}\right)$ and $\left(t_{0}, v_{0}\right)$, respectively, on $J$, we have

$$
d(u(t), v(t)):=\|u(t)-v(t)\| \leq r\left(t, t_{0}, w_{0}\right)
$$

provided $d\left(u_{0}, v_{0}\right) \leq w_{0}$. 
Now we can get the global existence of the solution of FDDIs. The fuzzy zero in $\mathbf{E}^{n}$ is denoted by $\hat{0}$.

Theorem 4.2 Assume that $F$ and $\alpha(x)$ obey the conditions to assure local solutions of FDDI (1), and for any $(t, u) \in \mathbf{R}_{+} \times C_{0}$,

$$
D(F(t, u), \hat{0}) \leq g(t,\|u\|)
$$

where $g \in C\left[\mathbf{R}_{+}^{2}, \mathbf{R}_{+}\right], g(t, w)$ is nondecreasing in $w$ for each $t \in \mathbf{R}_{+}$. Suppose that the solutions of

$$
w^{\prime}=g(t, w), \quad w\left(t_{0}\right)=w_{0} \geq 0,
$$

exists for $t \geq t_{0}$. Then the largest interval of existence of any solutions $x\left(t_{0}, \phi\right)$ of FDDI (1) is $\left[t_{0},+\infty\right)$.

Proof Let $x\left(t_{0}, \phi\right)(t)$ be a solution of (1) existing on some interval $\left[t_{0}-\tau, \alpha\right)$, where $t_{0}<\alpha$. Now suppose that $\alpha$ cannot be increased and we will get a contradiction.

Define on $\left[t_{0}-\tau, \alpha\right], m(t)=\left\|x\left(t_{0}, \phi\right)(t)\right\|$. Then $m_{t}=\left\|x_{t}\left(t_{0}, \phi\right)\right\|$. It follows that

$$
\begin{aligned}
D^{+} m(t):= & \limsup _{h \rightarrow 0} \frac{1}{h}[m(t+h)-m(t)] \\
\leq & \limsup _{h \rightarrow 0} \frac{1}{h}\left\|x\left(t_{0}, \phi\right)(t+h)-x\left(t_{0}, \phi\right)(t)\right\| \\
\leq & \sup _{f \in\left[F\left(t, x_{t}\right)\right]_{\alpha(x)}} \limsup _{h \rightarrow 0} \frac{1}{h}\left\|\frac{x(t+h)-x(t)}{h}-f\right\| \\
& +\sup _{e \in[F(t, 0)]_{\alpha(x)}} \limsup _{h \rightarrow 0}\|e\|+\sup _{e \in[F(t, 0)]_{\alpha(x), f \in\left[F\left(t, x_{t}\right)\right]_{\alpha(x)}}\|f-e\| .}
\end{aligned}
$$

Now the condition (*) implies that

$$
D^{+} m(t) \leq g\left(t,\left\|x_{t}\left(t_{0}, \phi\right)\right\|\right)=g\left(t,\left|m_{t}\right|_{0}\right), \quad t_{0} \leq t<\alpha
$$

Choosing suitable $\left|m_{t}\right|_{0}=\left\|\phi_{0}\right\|_{0} \leq w_{0}$ and letting $v(t)=0$, it follows from Lemma 4.1 that

$$
d\left(x\left(t_{0}, \phi\right)(t), 0\right)=\left\|x\left(t_{0}, \phi\right)(t)-0\right\| \leq r\left(t, t_{0}, w_{0}\right), \quad t_{0} \leq t<\alpha .
$$

Since $r^{\prime}=g(t, r) \geq 0$ and $r\left(t, t_{0}, w_{0}\right)$ is nondecreasing in $t$, we get

$$
d\left(x\left(t_{0}, \phi\right)_{t}, 0\right)=\left\|x\left(t_{0}, \phi\right)_{t}-0\right\| \leq r\left(t, t_{0}, w_{0}\right), \quad t_{0} \leq t<\alpha .
$$

For any $t_{1}$ and $t_{2}$ with $t_{0}<t_{1}<t_{2}<\alpha$, one can get

$$
\begin{aligned}
d\left(x\left(t_{0}, \phi\right)\left(t_{1}\right), x\left(t_{0}, \phi\right)\left(t_{2}\right)\right) & =\left\|x\left(t_{0}, \phi\right)\left(t_{2}\right)-x\left(t_{0}, \phi\right)\left(t_{1}\right)\right\| \\
& \leq \int_{t_{1}}^{t_{2}} g\left(s,\left\|x_{s}\left(t_{0}, \phi\right)\right\|\right) d s .
\end{aligned}
$$


By the nondecreasing property of $g$ in $w$, we have

$$
\begin{aligned}
d\left(x\left(t_{0}, \phi\right)\left(t_{1}\right), x\left(t_{0}, \phi\right)\left(t_{2}\right)\right) & \leq \int_{t_{1}}^{t_{2}} g\left(s,\left\|r\left(s, t_{0}, w_{0}\right)\right\|\right) d s \\
& =r\left(t_{2}, t_{0}, w_{0}\right)-r\left(t_{1}, t_{0}, w_{0}\right) .
\end{aligned}
$$

Letting $t_{1}, t_{2} \rightarrow \alpha$, the foregoing relation shows that $\lim _{t \rightarrow \alpha^{-}} x\left(t_{0}, \phi\right)(t)$ exists, because of Cauchy's criteria for convergence. Let $x\left(t_{0}, \phi\right)(\alpha)=\lim _{t \rightarrow \alpha^{-}} x\left(t_{0}, \phi\right)(t)$ and $\psi=x_{\alpha}\left(t_{0}, \phi\right)$. Then one can take $\psi$ as a new initial function of FDDI (1) at $t_{0}=\alpha$. By the assumption of the local existence of the solutions of FDDI (1), there exists a solution $x(\alpha, \psi)$ of (1) on some interval $[\alpha-\tau, \alpha+a], a>0$. This means that $x\left(t_{0}, \phi\right)$ could be continuously extended beyond $\alpha$, which is contrary to the assumption that $\alpha$ cannot be increased. This completes the proof.

Corollary 4.3 Suppose the assumptions of Theorem 4.2 hold and in addition, all the solutions $w\left(t, t_{0}, w_{0}\right)$ of

$$
w^{\prime}=g(t, w), \quad w\left(t_{0}\right)=w_{0} \geq 0,
$$

are bounded on $\left[t_{0},+\infty\right)$, then the solution $x\left(t_{0}, \phi\right)(t)$ of FDDI (1) tends to a finite limit as $t \rightarrow+\infty$.

Proof By the boundedness of $w\left(t, t_{0}, w_{0}\right)$, one can see that $\lim _{t \rightarrow \infty} w\left(t, t_{0}, w_{0}\right)$ is finite, which means that, for a given $\epsilon>0$, there exists a $t_{1}>t_{0}$ such that, for any $t>t_{1}$,

$$
0 \leq r\left(t, t_{0}, w_{0}\right)-r\left(t_{1}, t_{0}, w_{0}\right)<\epsilon \text {. }
$$

Since

$$
d\left(x\left(t_{0}, \phi\right)(t), x\left(t_{0}, \phi\right)\left(t_{1}\right)\right) \leq r\left(t, t_{0}, w_{0}\right)-r\left(t_{1}, t_{0}, w_{0}\right)<\epsilon
$$

we can see that $\lim _{t \rightarrow \infty} x\left(t_{0}, \phi\right)(t)$ exists and is finite. This completes the proof.

The trajectory $x(t)$ of FDDI (1) is often required to be constrained in a set $K$ rather than $\mathbf{R}^{n}$, for example, a fuzzy control system, which makes it necessary to discuss the viability of the trajectories of FDDIs.

Given a subset $K \subset \mathbf{R}^{n}$, the solution for the differential inclusion

$$
x^{\prime}(t) \in F(t, x(t)), \quad x\left(t_{0}\right)=x_{0}, \quad x_{0} \in K
$$

is said to be a viable trajectory on $\left[t_{0}, T\right)$ if $x(t) \in K$ for all $t \in\left[t_{0}, T\right)$.

Next we present a global time dependent viability theorem for FDDI (1) on $\left[t_{0},+\infty\right)$. Without loss of generality, we let $t_{0}=0, J_{0}=(-\infty, 0]$, and $C_{0}=C\left(J_{0}, \mathbf{R}^{n}\right)$ in the following theorem.

Theorem 4.4 Let $F: J_{0} \times C_{0} \rightarrow \mathbf{E}^{n}$ be a fuzzy map such that the corresponding function $F(t, x, y): J_{0} \times C_{0} \times \mathbf{R}^{n} \rightarrow[0,1]$ of which is upper semicontinuous at $(t, x) \in \Omega$. Suppose that 
$\alpha: \mathbf{R}^{n} \rightarrow[0,1)$ is a lower semicontinuous function and $K(t)$ is a set-valued map with closed graph from $[0,+\infty)$ to $\mathbf{R}^{n}$. We assume further that the tangential condition holds, that is,

$$
\begin{gathered}
\forall t \geq 0, \forall \phi \text { such that } \phi(0) \in K(t) \text { and } \forall x \in K(t), \\
{[F(t, \phi)]_{1} \cap D K(t, \phi(0))(1) \neq \emptyset .}
\end{gathered}
$$

For any $t \geq 0$, let $\mathcal{K}(t)=\left\{\phi \in C_{0}: \phi(0) \in K(t)\right\}$. Then for all $\phi \in K(0)$, there exists a solution $x(\cdot)$ to the FDDI (1), which is viable in the sense that

$$
\forall t \geq 0, \quad x(t) \in K(t)
$$

Proof The proof of this theorem is routine. Similar to the construction in Theorem 3.8, let $\widetilde{F}(t, x)=\left\{y \in \mathbf{R}^{n}: F(t, x, y) \geq \alpha(x)\right\}$. Then we know that $\widetilde{F}$ is an upper semicontinuous set-valued map with nonempty compact convex values. Because of the tangential condition (TC), for any $t \geq 0, \phi \in \mathcal{K}(t)$ and $x(t) \in K(t)$, we have $\widetilde{F}(t, \phi)=[F(t, \phi)]_{\alpha(x)}$ and so $\widetilde{F}(t, \phi) \cap D K(t, \phi(0))(1) \neq \emptyset$. Thus, one can complete the proof with the same routine of Theorem 4.7.1 in [41]. This completes the proof.

Taking $K(t)=\mathbf{R}^{n}$ in Theorem 4.4, we can get the property of the solution sets of the FDDIs (1).

Theorem 4.5 Let $F$ and $\alpha$ satisfy the assumptions in Theorem 4.4. Suppose that there exists a neighborhood D of $\left(t_{0}, \phi\right)$ such that $\bigcup_{(t, x) \in D}[F(t, x)]_{\alpha(x)}$ is compact in $\mathbf{R}^{n}$. Denote the set of solutions of the FDDIs $(1)$ by $\mathcal{T}_{\infty}(\phi)$. Then $\mathcal{T}_{\infty}(\phi)$ is an upper semicontinuous map from $C\left(J_{0}, \mathbf{R}^{n}\right)$ to the compact connected subsets of $C\left(\mathbf{R}, \mathbf{R}^{n}\right)$. Moreover, $\mathcal{T}_{\infty}(\phi)$ is $\sigma$-selectable, i.e., there exists a decreasing sequence of compact valued maps $\mathcal{T}_{n}$,

(i) $\forall n \geq 0, \mathcal{T}_{n}$ has a continuous selection;

(ii) $\forall \phi \in C\left(J_{0}, \mathbf{R}^{n}\right), \mathcal{T}_{\infty}(\phi)=\bigcap_{n} \mathcal{T}_{n}(\phi)$.

Proof Let $\widetilde{F}(t, x)=\left\{y \in \mathbf{R}^{n}: F(t, x, y) \geq \alpha(x)\right\}$. Then this theorem is the direct result of Theorem 2.2.1 and Corollary 2.2.4 in [41]. This completes the proof.

\section{Conclusions}

Let $\alpha(x)$ be constant, the results of this paper are constrained to the fuzzy differential inclusions in the sense of Hüllermeier. Generally, $F(t, x)$ is supposed to be upper semicontinuous. If, moreover, the boundedness assumption holds, with constants $b, M, T$. For any $\phi \in \operatorname{supp} \Phi$ and the inclusion $x^{\prime}(t) \in\left[F\left(t, x_{t}\right)\right]_{\alpha}, x_{0}=\phi$, we find that, when $\alpha$ ranges over $[0,1]$, by Theorem 6.2.3 in [13], the solution sets $\left\{x\left(x_{0}, \phi\right)(t)\right\}_{\alpha}$ are the $\alpha$-level sets of some fuzzy map $X\left(x_{0}, s\right)$, which is actually the solution of the following FDDE with fuzzy initial function $\Phi$ :

$$
\left\{\begin{array}{l}
x^{\prime}(t)=F\left(t, x_{t}\right), \\
x_{t_{0}}=\Phi, \quad \Phi \in C\left(J_{0}, \mathbf{E}^{n}\right) .
\end{array}\right.
$$

Therefore, the results presented in this paper extended the results in [11] and [13] to timevarying fuzzy delay differential inclusions. 


\section{Competing interests}

The authors declare that they have no competing interests.

\section{Authors' contributions}

All authors contributed equally to the writing of this paper. All authors read and approved the final manuscript.

\section{Author details}

${ }^{1}$ State Key Laboratory of Oil and Gas Reservoir Geology and Exploitation, Southwest Petroleum University, Xindu Road 8 in Xindu District, Chengdu, China. ${ }^{2}$ School of Science, Southwest Petroleum University, Xindu Road 8 in Xindu District, Chengdu, China. ${ }^{3}$ Department of Mathematics, Sichuan University, South Section 28, First Ring Road, Chengdu, China.

\section{Acknowledgements}

This paper is supported by the National Science Fund for Distinguished Young Scholars of China (51125019), Scientific Research Fund of Sichuan Provincial Education Department (13ZB0203) and the Natural Science Fund of Southwest Petroleum University (2012XJZ030). The authors are grateful to the editor and the referees for their valuable comments and suggestions.

\section{Received: 18 January 2014 Accepted: 26 March 2014 Published: 09 Apr 2014}

\section{References}

1. Puri, M, Ralescu, D: Differential and fuzzy functions. J. Math. Anal. Appl. 91, 552-558 (1983)

2. Kaleva, O: Fuzzy differential equations. Fuzzy Sets Syst. 24, 301-317 (1987)

3. Kaleva, O: The Cauchy problem for fuzzy differential equations. Fuzzy Sets Syst. 35, 389-396 (1990)

4. Kaleva, O: Nonlinear iteration semigroup of fuzzy Cauchy problem. Fuzzy Sets Syst. 209, 104-110 (2012)

5. Seikkala, S: On the fuzzy initial value problem. Fuzzy Sets Syst. 24, 319-330 (1987)

6. Chen, B, Liu, X: Reliable control design of fuzzy dynamical systems with time-varying delay. Fuzzy Sets Syst. 146 349-374 (2000)

7. Bede, B, Rudas, IJ, Bencsik, AL: First order linear fuzzy differential equations under generalized differentiability. Inf. Sci. $177,1648-1662(2007)$

8. Wu, C, Song, S, Lee, ES: Approximate solutions, existence and uniqueness of the Cauchy problem of fuzzy differential equations. J. Math. Anal. Appl. 202, 629-644 (1996)

9. Agarwal, RP, Lakshmikantham, V, Nieto, JJ: On the concept of solution for fractional differential equations with uncertainty. Nonlinear Anal. TMA 72, 2859-2862 (2010)

10. Diamond, P: Brief note on the variation of constants formula for fuzzy differential equations. Fuzzy Sets Syst. 129, 65-71 (2002)

11. Hüllermeier, E: An approach to modeling and simulation of uncertain dynamical systems. Int. J. Uncertain. Fuzziness Knowl.-Based Syst. 5, 117-137 (1997)

12. Lakshmikantham, V, Gnana Bhaskar, T, Vasundhara Devi, J: Theory of Set Differential Equations in Metric Spaces. Cambridge Scientific Publishers, Cambridge (2006)

13. Lakshmikantham, V, Mohapatra, RN: Theory of Fuzzy Differential Equations and Inclusions. Taylor \& Francis, London (2003)

14. Aubin, JP: Mutational and Morphological Analysis: Tools for Shape Evolution and Morphogenesis. Birkhäuser, Basel (1999)

15. Aubin, JP: Viability Theory. Birkhäuser, Basel (1991)

16. Diamond, $\mathrm{P}$, Watson, $\mathrm{P}$ : Regularity of solution sets for differential inclusions quasi-concave in a parameter. Appl. Math. Lett. 13, 31-35 (2000)

17. Bede, B, Gal, SG: Generalizations of the differentiability of fuzzy-number-valued functions with applications to fuzzy differential equations. Fuzzy Sets Syst. 151, 581-599 (2005)

18. Li, J, Zhao, A, Yan, J: The Cauchy problem of fuzzy differential equations under generalized differentiability. Fuzzy Sets Syst. 200, 1-24 (2012)

19. Khastan, A, Nieto, JJ, Rodriquez-Lopez, R: Variation of constant formula for first order fuzzy differential equations. Fuzzy Sets Syst. 177, 20-33 (2011)

20. Khastan, A, Nieto, JJ: A boundary value problem for second order fuzzy differential equations. Nonlinear Anal. TMA 72, 3583-3593 (2010)

21. Zhang, D, Feng, W, Zhao, Y, Qiu, J: Global existence of solutions for fuzzy second-order differential equations under generalized H-differentiability. Comput. Math. Appl. 60, 1548-1556 (2010)

22. Chen, M, Wu, C, Xue, X, Liu, G: On fuzzy boundary value problems. Inf. Sci. 178, 1877-1892 (2008)

23. Chen, $M, F u, Y, X u e, X, W u, C$ : Two-point boundary value problems of undamped uncertain dynamical systems. Fuzzy Sets Syst. 159, 2077-2089 (2008)

24. Liu, B: Fuzzy process, hybrid process and uncertain process. J. Uncertain Syst. 2(1), 3-16 (2008)

25. Qin, Z, Li, X: Option pricing formula for fuzzy financial market. J. Uncertain Syst. 2(1), 17-21 (2008)

26. Zhu, Y: Uncertain optimal control with application to a portfolio selection model. Cybern. Syst. 41(7), 535-547 (2010)

27. Choudary, ADR, Donchev, T: On Peano theorem for fuzzy differential equations. Fuzzy Sets Syst. 177, 93-94 (2011)

28. Nieto, JJ: The Cauchy problem for continuous fuzzy differential equation. Fuzzy Sets Syst. 102, $259-262$ (1999)

29. Filippov, AF: Differential equations with discontinuous right-hand side. Am. Math. Soc. Transl. 42(2), 199-231 (1964)

30. Baidosov, VA: Fuzzy differential inclusions. J. Appl. Math. Mech. 54(1), 8-13 (1990)

31. Dordan, O: Modelling fuzzy control problems with toll sets. Set-Valued Anal. 8, 85-99 (2000)

32. Zhu, Y, Rao, L: Differential inclusions for fuzzy maps. Fuzzy Sets Syst. 112, 257-261 (2000)

33. Lupulescu, V: On a class of fuzzy functional differential equations. Fuzzy Sets Syst. 160(11), 1547-1562 (2012)

34. Guo, M, Peng, X, Xu, Y: Oscillation property for fuzzy delay differential equations. Fuzzy Sets Syst. 200, 25-35 (2012)

35. Malinowski, MT: Itô type stochastic fuzzy differential equations with delay. Syst. Control Lett. 61, 692-701 (2012)

36. Kloeden, PE, Lorenz, T: Fuzzy differential equations without fuzzy convexity. Fuzzy Sets Syst. 230(1), 65-81 (2013) 
37. Michael, E: Continuous selections I. Ann. Math. 63, 361-381 (1956)

38. Aubin, JP, Frankowska, H: Set-Valued Analysis. Systems and Control: Foundations and Applications. Birkhäuser Boston, Boston (1990)

39. Hale, JK, Lunel, S: Introduction to Functional Differential Equations. Springer, New York (1993)

40. Yannelis, NC, Prabhakar, ND: Existence of minimal elements and equilibria in linear topological spaces. J. Math. Econ. 12, 233-245 (1983)

41. Aubin, JP, Cellina, A: Differential Inclusions. Springer, Berlin (1984)

10.1186/1687-1847-2014-108

Cite this article as: Min et al.: Existence of local and global solutions of fuzzy delay differential inclusions. Advances in Difference Equations 2014, 2014:108

Submit your manuscript to a SpringerOpen ${ }^{\circ}$ journal and benefit from:

- Convenient online submission

- Rigorous peer review

- Immediate publication on acceptance

- Open access: articles freely available online

- High visibility within the field

- Retaining the copyright to your article 